\title{
Dynamics of Public Opinions in an Online and Offline Social Network
}

\author{
Yucheng Dong, Zhaogang Ding, Francisco Chiclana, Enrique Herrera-Viedma
}

\begin{abstract}
Internet technology, the public opinions with big data will rapidly emerge in an online-offline social network, and an inefficient management of public opinions often will lead to the security crisis for either firms or governments. To unveil the interaction mechanism among a large number of agents between the online and offline social networks, in this paper we propose the public opinion dynamics model in an online-offline social network context. Next, in the theory aspect we investigate the analytical conditions to form a consensus in the public opinion dynamics model. Furthermore, we conduct the extensive simulations to investigate how the online agents impact the dynamics of public opinion formation, and unfold that the online agents shorten the steady-state time, decrease the number of opinion clusters, and smoothen the opinion changes in the opinion dynamics. The increase in the size of the online agents often enhances these effects. The results in this paper can provide a basis for the management of the public opinions in the Internet age.
\end{abstract}

Index Terms - opinion dynamics, social network, consensus, security, online and offline context, big data.

\section{INTRODUCTION}

$\mathrm{T}_{\mathrm{r}}^{\mathrm{s}}$ The Internet has been increasingly important for social networking. In the last decade, lots of online social networks have emerged, such as WeChat, Facebook, Twitter, and LinkedIn. People build their own friendship networks, and share their opinions, insights, information, experiences, and perspectives with each other in these networks [1], [37]. When these online social networks facilitate that the public can express their opinions regarding different issues such as politics, products and events, an important challenge is to deal with the management of public opinions. An inefficient management of public opinions often will lead to security crisis for either firms or governments.

Opinion dynamics is closely related to management of public opinions and a research tool widely used to investigate the opinion evolution in many collective phenomena. Some opinion dynamics models based on different communication regimes had been proposed. French [2] formulated a model on how persons' opinions are affected by the opinions of other persons with whom they are in direct communication. The subsequent work by DeGroot [3], [4] revaled that French's model of opinion change is special case of a more general model, and in DeGroot model, there is an interesting connection to the Delphi technique for pooling opinions of experts. Further, there is an interesting variation of DeGroot model developed by Friedkin and Johnsen [5], [6], and thus DeGroot

- Y.Dong is with School of Business, Sichuan University, Chengdu, Sichuan Province, 610065, P.R.China. E-mail:ycdong@scu.edu.cn.

- Z.Ding is with School of Business, Sichuan University, Chengdu, Sichuan Province, 610065, P.R.China. E-mail:dzgzhaogang@stu.scu.edu.cn.

- F. Chiclana is with Centre for Computational Intelligence, Faculty of Technology, De Montfort University, Leicester, UK .E-mail: chiclana@dmu.ac.uk.

- E. Herrera-Viedma is with Department of Computer Science and Artificial Intelligence, University of Granada, Granada, Spain, and also as visiting professor with Department of Electrical and Computer Engineering, Faculty of Engineering, King Abdulaziz University, Jeddah, Saudi Arabia. Email:viedma@decsai.ugr.es. model is a special case of Friedkin and Johnsen model. While the bounded confidence model is a pervasive nonlinear model, and this model introduced by Deffuant and Weisbuch [7] as well as the one by Hegselmann and Krause are rather similar [8]. Following the DW and HK models, some interesting extended studies had been conducted [9], [10], [11], [12], [13]. In recent years, some opinion dynamics models have been built based on social networks[14],[15],[16],[17], complex networks[18],[19],[20], dynamic networks[21],[22],[23],[24] and super-network [25].

However, many online social networks have emerged; a lot of people still only obtain information and exchange their opinions in offline social networks (e.g. face to face). For example, in China, the number of people who can access the Internet is close to 700 million, which implies that about half of the population in China can obtain information and express their opinions only via the traditional approach. Paying attention to previous studies they mainly focus on the online social network (e.g., [26], [27]). The interactions of people in the online social network and offline social network are different. Specially, an agent in the online social network makes friends with others who has similar interests (opinions, insights, experiences, or perspectives), and she/he also has friends in the offline social network. However, an agent in the offline social network gets to know others and makes friends mainly by his friends' friends. Meanwhile, there is the interaction between the online social network and offline social network. The public opinion in the online social network can trigger the collective action in the offline social network, and the collective action in the offline social network may become more severe because of the public opinion evolution in the online social network. Therefore, an important challenge for analysts is how to manage the dynamics of public opinions in the online and offline social network.

The aim of this study is not only to build the theoreti- 
cal foundations for the management of the public opinions, and but also unveil the interaction mechanism among a large number of agents between the online and offline social networks through extensive agent-based simulations and analyses. The remainder of this paper is arranged as follows. Section 2 introduces a basic description of graphs and opinion dynamics. Section 3 then proposes the public opinion dynamics model in an online and offline social network. Next, Section 4 provides the conditions to form a consensus in the proposed model via the theoretical analysis. Subsequently, Section 5 reveals some properties of the proposed model by simulation experiments. Finally, Section 6 presents the concluding remarks.

\section{Preliminaries}

This section introduces the basic knowledge regarding graphs, DeGroot model and bounded confidence model in opinion dynamics to develop our proposal.

\subsection{Graphs}

The basic definitions and notations regarding graphs can be found in [28], [29], [30] as Definitions 1-6.

Definition 1. A unidirectional graph is defined by $G(V, E)$, where $V=\left\{v_{1}, v_{2}, \ldots, v_{n}\right\}$ is a set of nodes, and $E$ is a set of pairs of elements of $V$ called edges. In this paper the sets $E$ and $V$ are assumed to be finite and $V$ is assumed to be nonempty.

Definition 2. The adjacency matrix $A=\left(a_{i j}\right)_{n \times n}$ of the unidirectional graph $G(V, E)$ is the zero-one matrix with 1 as its $(i, j)$ th entry when there is an edge between $v_{i}$ and $v_{j}$; Otherwise its $(i, j)$ th entry is 0 , i.e.,

$$
a_{i j}=\left\{\begin{array}{l}
1,\left(v_{i}, v_{j}\right) \in E \\
0,\left(v_{i}, v_{j}\right) \notin E
\end{array} .\right.
$$

Clearly, the adjacency matrix $A=\left(a_{i j}\right)_{n \times n}$ of the unidirectional graph $G(V, E)$ is symmetrical.

Definition 3. The degree of a node in a graph is the number of edges connected to that node.

Definition 4. A sequence of edges $\left(v_{i_{1}}, v_{i_{2}}\right)$, $\left(v_{i_{2}}, v_{i_{3}}\right), \ldots,\left(v_{i_{n-1}}, v_{i_{n}}\right)$ in a unidirectional graph $G(V, E)$ is called a path.

Definition 5. A unidirectional graph $G(V, E)$ is called connected if there is path between any two nodes. Otherwise, it is called disconnected.

Definition 6. The maximal connected subgraph of $G(V, E)$ is called its component. If there is only one component in a graph, the graph is connected; if there is more than one component, the graph is disconnected.

\subsection{Opinion dynamics}

Opinion dynamics describes the process of forming opinions among a large group of agents who continuously update their opinions based on the established rules, leading to a consensus or dissent in the final stage.

Let $\{1,2, \ldots, n\}$ be a set of the agents. Let $x_{i}^{t} \in R$ be the opinion of agent $i$ at time $t(t=0,1,2, \ldots)$, and thus $X^{t}=\left(x_{1}^{t}, x_{2}^{t}, \ldots, x_{n}^{t}\right)^{T} \in R^{n}$ be the opinion profile at time $t$. We introduce the DeGroot model and the bounded confidence model in details as follows.

(1) DeGroot model

The DeGroot mode has been proposed in [3], and is called the classical model in the opinion dynamics. Let $b_{i j}$ be the weight that agent $i$ gives to the agent $j$, where $b_{i j} \geq 0$ and $\sum_{j} b_{i j}=1$.

Then, the evolution of opinions of agent $i$ can be described by

$$
x_{i}^{t+1}=b_{i 1} x_{1}^{t}+b_{i 2} x_{2}^{t}+\ldots+b_{i n} x_{n}^{t} .
$$

Equation (2) can be compactly written as

$$
X^{t+1}=B \times X^{t},
$$

where $B=\left(b_{i j}\right)_{n \times n}$, and $B$ doesn't change with time or with opinions. Clearly, the DeGroot mode is a linear model in the opinion dynamics.

(2) The Hegselmann and Krause Model

The bounded confidence model assumes that each agent solely communicates with the agents who hold similar opinions and ignores the agents that have sufficiently different opinions. The earliest bounded confidence models have been introduced independently by Deffuant and Weisbuch [7] and by Hegselmann and Krause [8]. The two bounded confidence models are called the DW model and the HK model, respectively. In the HK model, agents synchronously update their opinions by averaging all opinions in their confidence sets; in the DW model, agents follow a pairwise-sequential updating mechanism. In this section, we briefly introduce the HK bounded confidence model. Note that if we adopt the DW model as the basis of our study, similar results will be obtained.

Let $\varepsilon$ be the homogeneous bounded confidence level of the agents. The process of the HK model consists of three steps as follows:

The first step is to determine of the confidence set. The confidence set $I\left(i, X^{t}\right)$ of the agent $i$ at time $t$ is determined as:

$$
I\left(i, X^{t}\right)=\left\{j|| x_{i}^{t}-x_{j}^{t} \mid \leq \varepsilon\right\} .
$$

Then, the second step is to calculate of the weights 
that one agent assigns to other agents. Let $w_{i j}^{t}$ be the weight of agent $i$ assigns to agent $j$ at time $t$, i.e.,

$$
w_{i j}^{t}=\left\{\begin{array}{ll}
1 / \# I\left(i, X^{t}\right), & j \in I\left(i, X^{t}\right) \\
0, & j \notin I\left(i, X^{t}\right)
\end{array},\right.
$$

where \#I $\left(i, X^{t}\right)$ is the cardinality of the set $I\left(i, X^{t}\right)$.

Finally, the third step is to determine the updated opinions for each agent. The updated opinion $x_{i}^{t+1}$ is modeled as a weighted arithmetic mean of opinions in the confidence set, i.e.,

$$
x_{i}^{t+1}=\sum_{j=1}^{N} w_{i j}^{t} x_{j}^{t} .
$$

Compared with the linear DeGroot model, the HK model is a pervasive nonlinear model, in which the weights depend on the evolution of opinions.

\section{MODELING PUBLIC OPINION DYNAMICS IN AN ONLINE-OFFLINE NETWORK}

In the proposed model, all agents and their relationships in the social network $S$ which are modeled by a unidirectional graph $G(V, E)$, where $V$ is the set of the agents in the social network, $E$ is the set of edges which indicate their relationships in the social network. All the agents in the social network $S$ are divided into two types: the online agents and the offline agents. For notational simplicity, let $V^{\text {on }}$ be the set of online agents, and let $V^{\text {off }}$ be the set of offline agents, where $V^{o n} U V^{\text {off }}=V$ and $V^{o n} \cap V^{o f f}=\varnothing$.

An offline agent can only communicates with her/his neighbour(s) in the social network. Therefore, we argue that the DeGroot model provides the potential to model the opinion communication mechanism, and thus we propose Hypothesis 1:

Hypothesis 1: In the public opinion dynamics the communication mechanism among the offline agents can be modelled by the DeGroot model.

On the other hand, the information and communication technologies facilitate that the online agents express their opinions, and an online agent will be influenced by the other online agents whose opinions are similar. Therefore, we argue that the HK bounded confidence model is a suitable tool to model the opinion communication mechanism among online agents, and thus we propose Hypothesis 2:

Hypothesis 2: In the public opinion dynamics the communication mechanism among the online agents can be modelled by the HK bounded confidence model.

In additional, there are communications between online agents and offline agents via the neighbour relationships in the social network $S$. And based on Hypothesizes 1 and 2, we propose the following public opinion dynamics model.

For any offline agent $i \in V^{\text {off }}$, agent $i$ only communicates with her/his neighbour(s) in the social network $S$, thus the confidence set $I(i, t)$ of the offline agent $i$ at time $t$ is determined as:

$$
I(i, t)=\left\{j \mid a_{i j}=1, j \in V\right\} .
$$

For any online agent $i \in V^{o n}$, agent $i$ not only communicates with her/his neighbour(s) in the social network $S$, but also communicates with the agents whose opinions differ from her/his own no more than certain confidence level $\varepsilon$. Thus the confidence set $I(i, t)$ of the online agent $i$ at time $t$ is determined by two parts:

$$
\begin{gathered}
I_{1}(i, t)=\left\{j \mid a_{i j}=1, j \in V\right\}, \\
I_{2}(i, t)=I\left(i, x_{i}^{t}\right)=\left\{j|| x_{i}^{t}-x_{j}^{t} \mid \leq \varepsilon, j \in V^{o n}\right\},
\end{gathered}
$$

and $I(i, t)=I_{1}(i, t) \cup I_{2}(i, t)$. In some case, $I_{1}(i, t) \bigcap I_{2}(i, t) \neq \varnothing$.

The agent $i$ gives the trust $\alpha_{i} \in(0,1)$ to own opinion, and distributes $\left(1-\alpha_{i}\right)$ across the other agents in her/his confidence set $I(i, t)$. Then, the updated opinion $x_{i}^{t+1}$ is calculated as

$$
x_{i}^{t+1}=\alpha_{i} x_{i}^{t}+\frac{1-\alpha_{i}}{\# I(i, t)} \sum_{j \in I(i, t)} x_{j}^{t},
$$

where \#I(i,t) denotes the cardinality of the confidence set $I(i, t)$. If $I(i, t)$ is an empty set, then $x_{i}^{t+1}=x_{i}^{t}$.

Equation (10) can be compactly written as

$$
X^{t+1}=F(t) X^{t},
$$

where $F$ varies with the time and opinions.

\section{THEORETICAL FOUNDATIONS: CONSENSUS CONDITIONS}

In opinion dynamics, the consensus is a core research problem. In this section, we investigate the conditions to form a consensus in public opinion dynamics model described by Equations (7)-(11). First, the concept of the consensus is defined as Definition 7.

Definition 7 [8]. All agents form a consensus if for and $X^{0} \in R^{n}$ there exists $c \in R$ such that 
$\lim _{t \rightarrow \infty} x_{i}^{t}=c(i=1,2, \ldots, n)$. Then, $c$ is called the consensus opinion.

A nonnegative matrix $M$ with the property that $M \overrightarrow{1}=\overrightarrow{1}$, where $\overrightarrow{1}=(1,1, \ldots, 1)^{T}$, i.e., all its row sums are 1 , is defined to be a (row) stochastic matrix.

Let $M^{t}$ be the matrix power of $M$. If $\lim _{t \rightarrow \infty} M^{t}$ is a matrix of rank 1 , i.e. ,

$$
\lim _{t \rightarrow \infty} M^{t}=\overrightarrow{1} m,
$$

where $\overrightarrow{1}=(1,1, \ldots, 1)^{T}$ and $m=\left(m_{1}, m_{2}, \ldots, m_{n}\right)$, then we call $M$ an ergodic matrix [29] .

Before analysis the condition to form a consensus in public opinion dynamics model, we introduce Lemmas 1 and 2.

Lemma 1 (Wolfowitz) [30, 31]. Let $M_{1}, M_{2}, \ldots, M_{l}$ be a finite set of ergodic $n \times n$ matrices with the property that for each sequence $M_{i_{1}}, M_{i_{2}}, \ldots, M_{i_{j}}$ of positive length, the matrix product $M_{i_{j}} M_{i_{j-1}} \cdots M_{i_{1}}$ is ergodic. Then for each infinite sequence there exists a row vector $\theta=\left(\theta_{1}, \theta_{2}, \ldots, \theta_{n}\right)$ such that

$$
\lim _{j \rightarrow \infty} M_{i_{j}} M_{i_{j-1}}, \ldots, M_{i_{1}}=\overrightarrow{1} \theta,
$$

where $\overrightarrow{1}=(1,1, \ldots, 1)^{T}, \theta=\left(\theta_{1}, \theta_{2}, \ldots, \theta_{n}\right)$.

Lemma 2 [32]. Let $M_{1}, M_{2}, \ldots, M_{k}(k \geq 2)$ be nonnegative $n \times n$ matrices, and the diagonal elements of all of the $M_{i}$ are positive and let $\mu, \rho$ denote the smallest and largest of these, respectively. Then

$$
M_{1} M_{2} \cdots M_{k} \geq\left(\frac{\mu^{2}}{2 \rho}\right)^{k-1}\left(M_{1}+M_{2}+\cdots+M_{k}\right) .
$$

Based on Lemmas 1 and 2, we propose the consensus condition in public opinion dynamics model, as Theorems 1 and 2 .

Theorem 1. If the social network $S$ is connected, all agents can reach a consensus finally in public opinion dynamics model.

Proof: Since the social network $S$ is connected, the adjacency matrix $A$ of the social network $S$ must be irreducible matrix. According to Eqs.(7) and (8), for any finite time $t_{0}$, we have $F\left(t_{0}\right)$ is an irreducible and nonnegative matrix. In additional, for any agent $\alpha_{i} \in(0,1)$, and thus the diagonal elements of all of the $F\left(t_{0}\right)$ are positive. Hence $F\left(t_{0}\right)$ is a primitive matrix.
According to Equation 10, we have $F\left(t_{0}\right)$ is a stochastic matrix, and thus $F\left(t_{0}\right)$ is an ergodic matrix.

As we know, the product of two nonnegative matrices with positive diagonals is a matrix with the same properties and because the product of two stochastic matrices is stochastic. And thus for each matrix product $F\left(t_{0}\right) F\left(t_{0}-1\right) \cdots F(2) F(1)$ is a matrix with positive diagonals. Based on Lemma 2, for each matrix product $F\left(t_{0}\right) F\left(t_{0}-1\right) \cdots F(2) F(1)$ is an irreducible matrix, and thus each matrix product $F\left(t_{0}\right) F\left(t_{0}-1\right) \cdots F(2) F(1)$ is primitive, as a result, ergodic. Based on Lemma 1, we have

$$
\lim _{t \rightarrow \infty} F(t) F(t-1) \cdots F(2) F(1)
$$

is a matrix of rank 1 . Hence all agents in the social network $S$ can reach a consensus finally for any $X^{0} \in R^{n}$.

Furthermore, if the disconnected social network $S$ only has two components $S_{1}$ and $S_{2}$, and there are online agents in $S_{1}$ and $S_{2}$, respectively. $V_{1}^{o n}$ and $V_{2}^{o n}$ are the sets of the online agents in the social network $S_{1}$ and $S_{2}$. In this case, we propose the consensus condition as Theorem 2.

Theorem 2. Let $t_{0}$ be the finite time, for any $t \geq t_{0}$, if there exists

$$
\begin{aligned}
& \left(\bigcup_{i \in V_{1}^{o n}} I(i, t)\right) \cap V_{2}^{o n} \neq \varnothing \\
& \left(\bigcup_{i \in V_{2}^{o n}} I(i, t)\right) \cap V_{1}^{o n} \neq \varnothing,
\end{aligned}
$$

or

then all agents in the social network $S$ can reach a consensus finally in public opinion dynamics model.

Proof: We need the fact concerning the confidence set, considering the homogeneous bounded confidence level of the online agents, when the online agent $j \in I\left(i, X^{t}\right)$, we must have the online agent $i \in I\left(j, X^{t}\right)$.

There exists the finite time $t_{0}$, for any $t_{1} \geq t_{0}$, if

$$
\left(\bigcup_{i \in V_{1}^{o n}} I(i, t)\right) \cap V_{2}^{o n} \neq \varnothing \text {, }
$$

We must have

$$
\left(\bigcup_{i \in V_{2}^{o n}} I(i, t)\right) \cap V_{1}^{o n} \neq \varnothing
$$

and vice versa.

Since $S_{1}$ and $S_{2}$ is two components of $S$, and thus $S_{1}$ and $S_{2}$ are connected, respectively. For the finite time 
$t_{1} \geq t_{0}$, there exists

$$
\left(\bigcup_{i \in V_{1}^{o n}} I(i, t)\right) \cap V_{2}^{o n} \neq \varnothing,
$$

we must have

$$
\left(\bigcup_{i \in V_{2}^{o n}} I(i, t)\right) \cap V_{1}^{o n} \neq \varnothing .
$$

i.e., the agents $i$ and $j$ connect $S_{1}$ and $S_{2}$, and thus $F\left(t_{1}\right)$ is an irreducible nonnegative matrix. In additional, the diagonal elements of all of the $F\left(t_{1}\right)$ are positive, and thus $F\left(t_{1}\right)$ is primitive, as a result, ergodic.

For each matrix product $F\left(t_{1}\right) F\left(t_{1}-1\right) \cdots F\left(t_{0}\right)$ is a stochastic matrix with positive diagonals, based on Lemma 2, for each matrix product $F\left(t_{1}\right) F\left(t_{1}-1\right) \cdots F\left(t_{0}\right)$ is an irreducible nonnegative matrix. And thus $F\left(t_{1}\right) F\left(t_{1}-1\right) \cdots F\left(t_{0}\right)$ is an ergodic matrix. Based on Lemma 1, we have

$$
\lim _{t \rightarrow \infty} F(t) F(t-1) \cdots F\left(t_{0}+1\right) F\left(t_{0}\right)=\overrightarrow{1} \theta,
$$

where $\overrightarrow{1}=(1,1, \ldots, 1)^{T}, \theta=\left(\theta_{1}, \theta_{2}, \ldots, \theta_{n}\right)$.

In additional, we have

$$
\begin{aligned}
& \lim _{t \rightarrow \infty} F(t) F(t-1) \cdots F\left(t_{0}+1\right) F\left(t_{0}\right) F\left(t_{0}-1\right) \cdots F(1)-\overrightarrow{1} \theta F\left(t_{0}-1\right) \cdots F(1) . \\
& =\left(\lim _{t \rightarrow \infty} F(t) F(t-1) \cdots F\left(t_{0}+1\right) F\left(t_{0}\right)-\overrightarrow{1} \theta\right) F\left(t_{0}-1\right) \cdots F(1)
\end{aligned}
$$

For the finite time $t_{0}$, and thus $F\left(t_{0}-1\right) \cdots F(1)$ is a bounded function. Hence we have $\lim _{t \rightarrow \infty} F(t) F(t-1) \cdots F\left(t_{0}+1\right) F\left(t_{0}\right) F\left(t_{0}-1\right) \cdots F(1)=\overrightarrow{1} \theta F\left(t_{0}-1\right) \cdots F(1)$, where $\overrightarrow{1}=(1,1, \ldots, 1)^{T}, \theta=\left(\theta_{1}, \theta_{2}, \ldots, \theta_{n}\right)$.

Hence all agents in the social network $S$, for any $X^{0} \in R^{n}$, can reach a consensus finally.

From Theorem 2, we directly have Corollary 1.

Corollary 1: When there are several components in the disconnected social network $S$, and there are online agents in every component. If some online agents in different components can keep in touch with other online agents as time goes on, i.e., they are in each other's confidence sets as time goes on, then all agents in the disconnected social network $S$ can reach a consensus finally.

Theorem 1 shows that all agents can reach a consensus finally in a connected social network. Theorem 2 and Corollary 1 shows that online agents will avail the consensus formation in a disconnected social network.

\section{SimULATION EXPERIMENTS}

In this section, we focus on how the online agents impact the dynamics of public opinion formation by simulation experiments from the two aspects: The influences of online agents in the disconnected social network (see Section 5.1), and the influences of online agents in the connected social network (see Section 5.2).

Many real-lift social networks have been investigated by using social big data which is a collection of very huge data sets of social networks with great diversity (e.g., Paper database, Twitter, Facebook, LinkedIn and mobile social networks) [1], [33], [38]. These real social networks often have the following features:

(1) The small-world effect: Most pairs of nodes in most social network seem to be connected by a short path through the network.

(2) The degree distribution in some real social networks follows a power law.

Thus, we will construct the social networks with these properties in simulation experiments.

In the simulation experiments, there are $N$ agents and let $r=\left(\# V^{o n}\right) /(\# V)$ be the percentage of the online agents in all agents, where \# is the cardinality of the finite set. We randomly set $N \times r$ agents to be the online agents and the rest of the agents are offline agents. The initial opinions of all agents are uniformly random distribution in $[0,1]$, and the confidence level of the agents are no more than 0.3 in general. Otherwise, all agents can reach a consensus in HK model. The self-confidence level of the agents are homogeneous, and set $\alpha_{i}=0.5$ for all agents $i$ in this paper. In the simulation experiments, when $\left\|X^{t+1}-X^{t}\right\|_{1} \leq \delta$, we consider that the opinions of all agents reach the stable state, where $\|X\|_{1}=\sum_{i=1}^{N}\left|x_{i}\right|$. In this paper, we set $\delta=10^{-4}$. Meanwhile, let $x_{i}, x_{j}$ be the opinions of agents $i$ and $j$ when the opinions reach the stable state. We assign the agents $i$ and $j$ to a same cluster when $\left|x_{i}-x_{j}\right|<d$, and we set $d=10^{-2}$.

\subsection{The influences of online agents in the disconnected social network}

We investigate the influences of online agents in the disconnected social network based on five criteria, the steady-state time, the number of opinion clusters, the maximum opinion cluster size, and the number of pure online/offline opinion cluster.

(1) The steady-state time $T$ is defined as the mini- 
mum time it takes all agents' opinions to reach a stable state.

(2) The opinion clusters appear when the stable state is finally reached in opinion dynamics. The number of opinion clusters $S_{N C}$ is defined as the number of different opinion clusters among the agents in the stable state. Larger $S_{N C}$ values indicate more different opinions among the agents in the stable state. In particular, $S_{N C}=1$ indicates all agents reach a consensus finally.

(3) $S_{M N C}$ is defind as the maximum size of opinion cluster when the stable state is finally reached in opinion dynamics, and $S_{M N C}$ is a measure of the power of the majority. In this paper, let $S_{M N C}^{*}=S_{M N C} / N$.

(4) The pure online opinion cluster is defined as an opinion cluster in which all agents are online agents and the pure offline opinion cluster is defined as an opinion cluster in which all agents are offline agents. $S_{N P C}^{o n}$ and $S_{N P C}^{o f f}$ denote the number of pure online opinion cluster and pure offline opinion cluster in the stable state, respectively.

In the simulation experiments, we choose Erdôs \&Rényi (ER) random graph [34] $G_{N, p}$ to construct the social network. Specifically, take $N$ agents and connect each pair agents with probability $p$. As $p$ increases, the disconnected social network will change gradually to a connected one, where $p_{c} \approx \ln (N) / N$ is a sharp threshold for the connectedness of $G_{N, p}$, i.e. , if $p<(1-\gamma) p_{c}$, then a graph in $G_{N, p}$ will almost surely contain isolated agents, and thus be disconnected; If $p>(1-\gamma) p_{c}$, then a graph in $G_{N, p}$ will almost surely be connected, where $\gamma$ is positive real number and near to 0 . In additional, the degree distribution of $G_{N, p}$ is a Poisson distribution.

Then, using models (i.e., Equations (7)-(11)) proceeds with the evolution of opinions, obtaining the average $T$, $S_{N C}, S_{M N C}^{*}, S_{N P C}^{o n}$ and $S_{N P C}^{o f f}$ values from 1000 independent realizations. In the simulation experiments, we set that $N=200$ and $\varepsilon=0.15$.

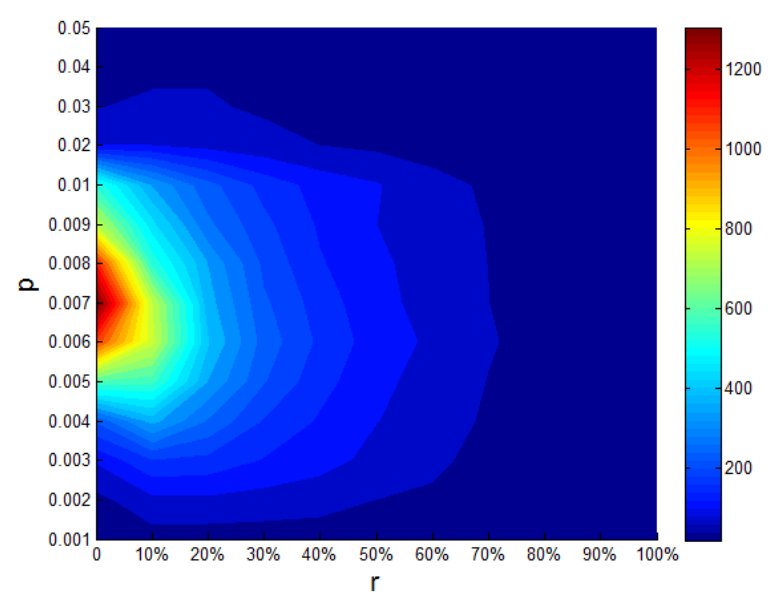

Fig. 1. The average $T$ values under different $p$ and $r$ values.

Fig. 1 reveals the impact of $p$ and $r$ on the steadystate time. As $r$ increases, the steady-state time $T$ decreases, which means that the more size of online agents is, the less time the stable state needs. Thus, the communication regime of online agents avails the stabilization of the opinion dynamics.

As $p$ increases, the steady-state time $T$ starts increasing, and then decreases. When $p$ is a small value (e.g. $0.001)$, the edges in the ER random network is fewer, and then the opinion dynamics stabilizes quickly because many agents do not communicate with others. When $p$ is large (e.g. 0.03), the social network is almost surely connected and the opinion dynamics stabilizes quickly because of numerous communications among the agents. However, when the $p$ value is between 0.005 and 0.01 , the social network changes its topology abruptly from a loose collection of small clusters to being dominated by a single giant cluster, and there are some cycles in the social network, leading that the steady-state time $T$ is longer than the above two situations.

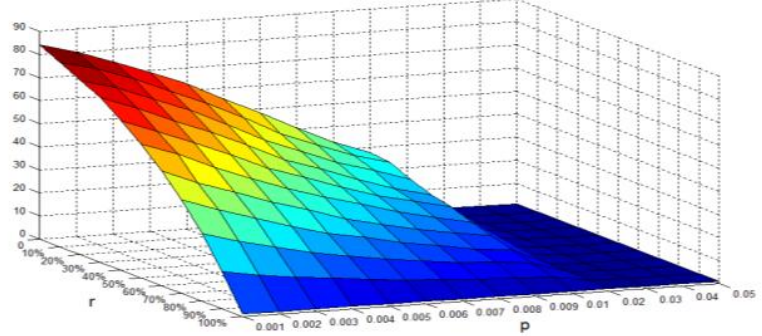

Fig. 2. The average $S_{N C}$ values under different $p$ and $r$ values.

Fig. 2 reveals the impact of $p$ and $r$ on opinion clusters. When $p \leq 0.02$, as $r$ increases, $S_{N C}$ decreases, 
which means that the larger size of online agents yields the fewer number of opinion clusters because the communication regime of online agents facilitates that many disconnected agents can communicate with each other. As $p$ further increases (e.g. $p>0.02$ ), the social network is almost surely connected, and thus the impact of $r$ on $S_{N C}$ is not evident.

For a fixed $r$, as $p$ increases, $S_{N C}$ starts decreasing, and then stabilizes. When $p$ is small, there are many disconnected agents in the social network, and thus the value of $S_{N C}$ is large. As $p$ increases, more agents are connected, so $S_{N C}$ decreases. When $p$ is large (e.g. 0.03), the social network is almost surely connected, $S_{N C}$ stabilizes due to all agents can reach a consensus easily. i.e. $S_{N C}=1$.

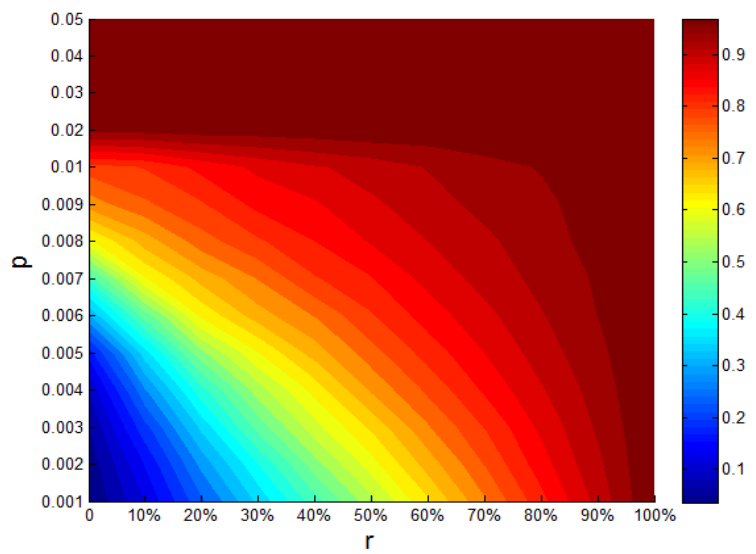

Fig. 3. The average $S_{M N C}^{*}$ values under different $p$ and $r$ values.

Fig. 3 reveals the impact of $p$ and $r$ on $S_{M N C}^{*}$. When $p \leq 0.02$, as $r$ increases, $S_{M N C}^{*}$ increases, i.e., the more size of online agents is, the larger the maximum opinion cluster size is. The communication regime of online agents facilitates that many disconnected agents in social network can cluster together. As $p(p \geq 0.02)$ further increases, the social network is almost surely connected, and thus all agents reach a consensus easily, Hence the impact of $r$ on $S_{M N C}^{*}$ is not evident in this situation.

For a fixed $r$, as $p$ increases, $S_{M N C}^{*}$ starts increasing, and then stabilizes. As $p$ increases from $p=0.001$ to $p=0.02$, more agents are connected and thus $S_{M N C}^{*}$ increases. When $p \geq 0.02$, the social network is almost surely connected, and thus $S_{M N C}^{*}$ stabilizes due to all agents can reach a consensus easily. i.e. $S_{M N C}^{*}=1$.

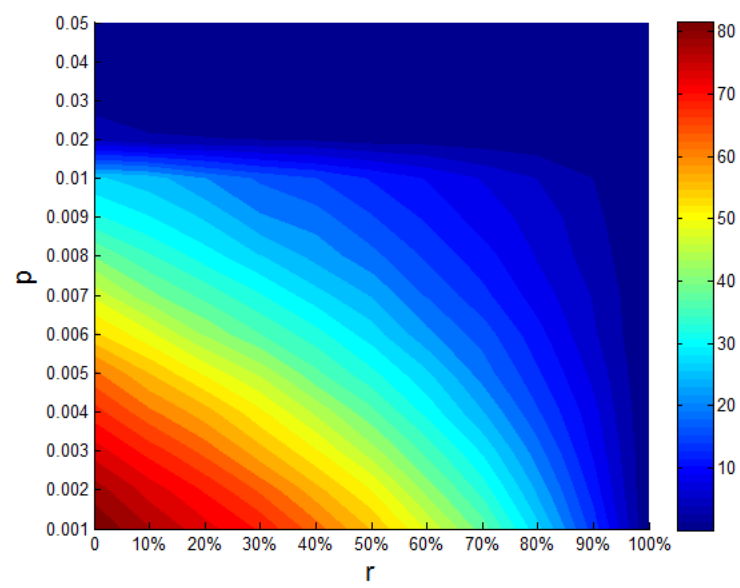

Fig. 4. The average $S_{N P C}^{\text {off }}$ values under different $p$ and $r$ values.

Fig. 4 reveals the impact of $p$ and $r$ on $S_{N P(\text {. }}^{o f f}$. $S_{N P C}^{o f f}$ highlights the lower left-hand corner of Fig. 4. When the values $p$ and $r$ are small, there are many offline opinion clusters. When $p \leq 0.02$, as $r$ increases, $S_{N P C}^{o f f}$ decreases, i.e., the more size of online agents is, the smaller the number of pure offline opinion cluster is. Because the online agents always attract some offline agents, and thus the number of pure offline opinion cluster decreases. As $p(p \geq 0.02)$ further increases, the social network is almost surely connected, and thus the impact of $r$ on $S_{N P C}^{o f f}$ is not evident.

For a fixed $r$, as $p$ increases, $S_{N P C}^{o f f}$ starts decreasing, and then stabilizes. As $p$ increases from $p=0.001$ to $p=0.02$, more agents are connected, and thus $S_{N P C}^{\text {off }}$ decreases. When $p \geq 0.02$, the social network is almost surely connected, and thus $S_{N P C}^{o f f}$ stabilizes due to all agents can reach a consensus easily. In this case, the value of $S_{N P C}^{\text {off }}$ is very small, and even the value is 0 .

However, we find that pure online clusters are hardly observed in the simulations, and this phenomenon implies that online agents can always attract a certain number of offline agents. The pure offline clusters are easily observed which implies that some of the offline agents could be isolated from society because of the development of the information and communication technologies.

\subsection{The influences of online agents in the connected social network}

In this section, we investigate the influences of online agents in a connected social network under different topology, such as the ER random network, the Watts \& Strogatz (WS) small world network [35] and the Barabási 
\& Albert (BA) scale-free network [36].

In the WS small world network, start with a ring lattice with $N$ agents in which every agent is connected to her/his first $K$ neighbors ( $K / 2$ on either side), and then randomly rewire each edge of the lattice with probability $p$ such that self-connections and duplicate edges are excluded. When $p$ is small, the WS small world network has a high clustering coeffcient, i.e., there are many circles of friends or acquaintances in which every member knows every other member.

In the BA scale-free network, starting with a small number $m_{0}$ of agents which are all connected to each other, at every time step we add a new agent with $m_{1}$ $\left(m_{1} \leq m_{0}\right.$ ) edges that link the new agent to $m_{1}$ different agents already present in the system, such that the probability to get linked to an agent is proportional to her/his degree. After $N-m_{0}$ time steps, there are $N$ agents in the network. The degree distribution of this BA scale-free network is a power-law distribution with the fixed power exponent 3 .

In the simulation experiments, the parameters regarding to three networks are listed in Table 1. The sizes in three networks are same, i.e. $N=200$, and the average degree in three networks are all about 6 , and we choose connected network in every time of simulation process to guarantee all agents can reach a consensus finally.

TABLE 1

THE PARAMETERS REGARDING TO THREE NETWORKS

\begin{tabular}{cc}
\hline \hline $\begin{array}{c}\text { The Topology of } \\
\text { network }\end{array}$ & Parameters \\
\hline ER random & $N=200, p=0.03$ \\
WS small world & $N=200, K=6, p=0.01$ \\
BA scale-free & $N=200, m_{0}=6, m_{1}=3$ \\
\hline \hline
\end{tabular}

Then, we pay attention to three criteria to investigate the influences of online agents in a connected social network, i.e., the consensus time, the maximum opinion changes, and the sum of the opinion changes.

(1) The consensus time $T^{c}$ is defined as the minimum time it takes all agents' opinions to reach a consensus.

(2) The $\operatorname{Moc}(t)$ is a measure of maximum opinion changes of all agents from one time instant to next in the opinion dynamics. i.e.,

$$
\operatorname{Moc}(t)=\left\|X^{t}-X^{t-1}\right\|_{\infty},
$$

where $\|X\|_{\infty}=\max \left\{\left|x_{1}\right|,\left|x_{2}\right|, \ldots,\left|x_{N}\right|\right\}, t=1,2, \ldots$.

(3) The $\operatorname{Soc}(t)$ is a measure of the sum of the opinion changes of all agents from one time instant to next in opinion dynamics. i.e.,

$$
\operatorname{Soc}(t)=\left\|X^{t}-X^{t-1}\right\|_{1}^{\prime}
$$

where $\|X\|_{1}=\sum_{i=1}^{N}\left|x_{i}\right|, t=1,2, \ldots$.

The simulation setup is the same with the former Section 5.1, using models (i.e., Equations (7)-(11)) proceeds with the evolution of opinions, obtaining the average $T^{c}$, $\operatorname{Moc}(t)$ and $\operatorname{Soc}(t)$ values from 1000 independent realizations.

Fig.5 shows that the consensus time in the ER random network, the WS small world network and the BA scale-free network, respectively. In the ER random network, as $r$ increases, $T^{c}$ starts increasing gently and then decreases. The results in the BA scale-free network are similar to the ER random network. However, $T^{c}$ decreases with $r$ increasing in the WS small world network because the online agents avail communications among agents who have long distance in the WS small world network. In addition, the higher confidence level of online agents makes consensus time decreasing evidently in the three networks, especially for lager $r$ values because the higher confidence level makes online agents' influence capacities to become strengthen.
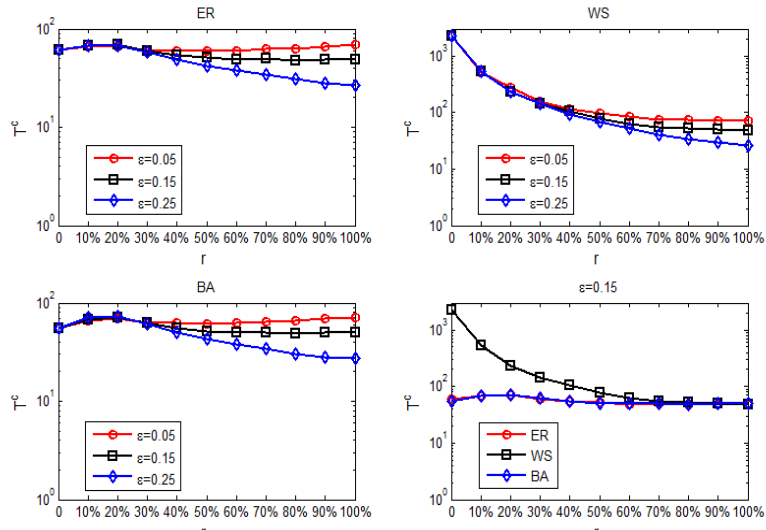

Fig. 5. The average $T^{c}$ values under different networks topologies and $r$ values

Although three networks have the same size and the same average degree in the simulation experiments, the ER random network and the BA scale-free network have less average shortest path length than the WS small world network, and the WS small world network has a higher clustering coeffcient than the ER random network and the BA scale-free network. Generally, the network which has a less average shortest path avails that all agents reach a consensus quickly, while the network which has a higher clustering coeffcient always leads to that all agents reach 
a consensus slowly. Thus, the consensus time in the WS creases, the differences among three networks decrease. small world network is always longer than the ER random network and the BA scale-free network. As $r$ in-
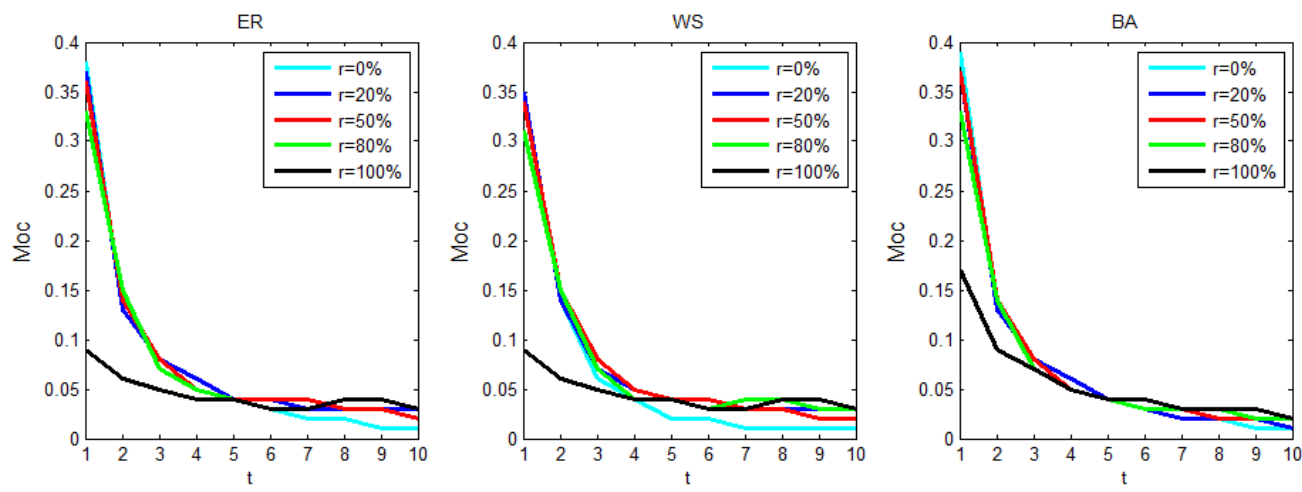

Fig. 6. The average $\operatorname{Moc}(t)$ values under different network topologies and $r$ values
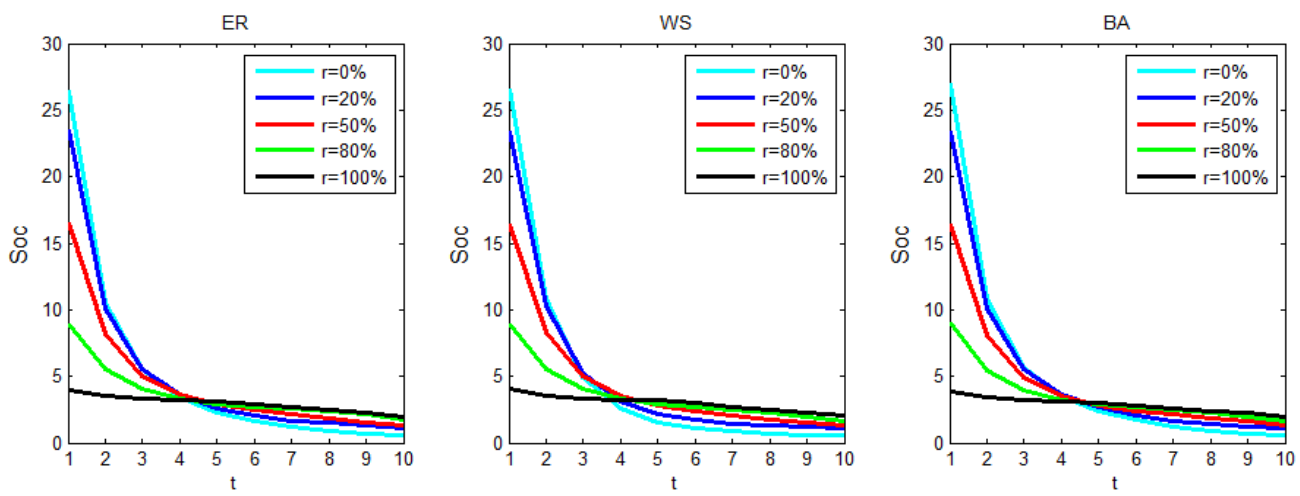

Fig.7. The average $\operatorname{Soc}(t)$ values under different network topologies and $r$ values

Fig.6 shows that the maximum opinion change of all agents in the ER random network, the WS small world network and the BA scale-free network, respectively. All agents reach a consensus finally in the connected network, and $\operatorname{Moc}(t)$ decreases gradually with the time going on. We only show $\operatorname{Moc}(t)$ from $t=1$ and $t=10$. In three networks, for a fixed value of $r, \operatorname{Moc}(t)$ decreases from $t=1$ and $t=10$. In other words, the opinions of all agents change rapidly in the first ten time instants. As $r$ increases, this trend will be alleviated especially for $r \geq 80 \%$. The results in the ER random network and the WS small world network are very similar because the shape of degree distribution of the WS small world network is similar to that of the ER random network, and the topologies of two networks are relatively homogeneous. While the BA scale-free network has a power-law degree distribution, when all agents are online agents, the average value of $\operatorname{Moc}(1)$ in the BA scale-free network is still more than in the ER random network and the WS small world network.

Fig. 7 shows that the sum of the opinion changes of all agents in the ER random network, the WS small world network and the BA scale-free network, respectively. Similar to $\operatorname{Moc}(t)$, we show $\operatorname{Soc}(t)$ from $t=1$ and $t=10$. In three networks, for a fixed value of $r$, Soc $(t)$ decreases from $t=1$ and $t=10$. In other words, $\operatorname{Soc}(t)$ varies very rapidly in the first ten time instants. As $r$ increases, this trend will be alleviated especially for $r \geq 80 \%$. In addition, the results of $\operatorname{Soc}(t)$ are very similar in the ER random network, the WS small world network and the BA scale-free network although the topologies of three networks are different.

\section{Conclusion}

In this paper, we propose the public opinion dynamics in an online and offline social network. The main contributions are as follows.

(1) We analyze the communication mechanisms among the online and offline agents, and propose the public opinion dynamics model in an online and offline social network.

(2) We present the analytical conditions to form a 
consensus in the public opinion dynamics model.

(3) Through the extensive simulation experiments, we unfold how the online agents impact the dynamics of public opinion formation.

In the future, we plan to work on the following two issues:

(1) We plan to make use of our proposed model to support the consensus reach process [38, 39, 40, 41, 42] which is a dynamic and iterative process guided by a moderator and composed by several rounds in which the individuals express, discuss and modify their opinions until reaching an agreed decision;

(2) The asynchronization or time-delayed is a very popular phenomenon in the evolution of real-life public opinions [43]. In the future, we plan to study the asynchronization phenomenon in the public opinion dynamics in an online and offline social network context and use real data to verify our proposed models.

\section{ACKNOWLEDGMENT}

This work was supported by the grants (Nos. 71171160 and 71571124) from NSF of China, the grant (No. skqy201606) from Sichuan University, the grant (No. TIN2013-40658-P) from the FEDER funds, and the grant (No. TIC-5991) from the Andalusian Excellence Project.

\section{REFERENCES}

[1] S. Yu, M. Liu, W. Dou, X. Liu, and S. Zhou, "Networking for Big Data: A Survey," IEEE Communications Surveys and Tutorials, in press, 2016

[2] J. R.P. French, "A formal theory of social power," Psychological Review 63,181-194, 1956.

[3] M.H.DeGroot, "Reaching a consensus," Journal of the American Statistical Association 69,118-121, 1974.

[4] R.L.Berger, "A necessary and sufficient condition for reaching a consensus using DeGroot's method," Journal of the American Statistical Association 76, 415-418, 1981.

[5] N.E. Friedkin, and E.C. Johnsen, "Social influence and opinions," Journal of Mathematical Sociology 15, 193-205, 1990.

[6] N.E. Friedkin \& E.C. Johnsen, "Social influence networks and opinion change," Advances in Group Processes 16, 1-29, 1999.

[7] G. Deffuant, D. Neau, F. Amblard, and G. Weisbuch, "Mixing beliefs among interacting agents," Advances in Complex Systems 3, 87-98, 2000.

[8] R. Hegselmann, and U. Krause, "Opinion dynamics and bounded confidence models, analysis, and simulation," Journal of Artificial Societies and Social Simulation 5, 2-34, 2002.

[9] J. Lorenz, "Consensus strikes back in the Hegselmann-Krause model of continuous opinion dynamics under bounded confidence," Journal of Artificial Societies and Social Simulation 9, 2006.

[10] S. Fortunato, V. Latora, A. Pluchino, and A. Rapisarda, "Vector opinion dynamics in a bounded confidence consensus model," International Journal of Modern Physics C 16, 1535-1551, 2005.
[11] H.M. Liang, Y.C. Dong, and C.C. Li, “Dynamics of uncertain opinion formation: An agent-based simulation," Journal of Artificial Societies and Social Simulation 19, 2016.

[12] Y.C. Dong, X. Chen, H.M. Liang, and C.C. Li, “Dynamics of linguistic opinions formation in bounded confidence model," Information Fusion 32, 52-61, 2016.

[13] H.M. Liang, C.C. Li, Y.C. Dong, and Y.P. Jiang, "The fusion process of interval opinions based on dynamic bounded confidence," Information Fusion 29,112-119, 2016

[14] Y. Eom, and D. Shepelyansky, “Opinion formation driven by PageRank node influence on directed networks," Physica A 436,707-715, 2015.

[15] Z. Liu, J.F. Ma, Y. Zeng, L. Yang, Q. Huang, and H. Wu, “On the control of opinion dynamics in social networks," Physica A 409,183198, 2014.

[16] C. Qian, J. Cao, J. Lu, and J. Kurths, “Adaptive Bridge control strategy for opinion evolution on social networks," Chaos 21, Article ID 025116, 2011.

[17] E. Kurmyshev, H.A. Juárez, and R.A. González-Silva, “Dynamics of bounded confidence opinion in heterogeneous social networks: Concord against partial antagonism," Physica A Statistical Mechanics \& Its Applications 390, 2945-2955, 2011.

[18] N. Masuda, "Opinion control in complex networks," New Journal of Physics 17, 033031, 2015.

[19] P.P. Li, D.F. Zheng, and P.M. Hui, "Dynamics of opinion formation in a small-world network," Physical Review E Statistical Nonlinear \& Soft Matter Physics 73, 88-101, 2006.

[20] M. Jalili, "Coevolution of opinion formation and network dynamics in complex networked systems," International Conference on Information Society, 2015.

[21] W. Ren, and R.W. Beard, Consensus of information under dynamically changing interaction topologies, American Control Conference 6, 4939-4944, 2004.

[22] F. Kuhn, R. Oshman, and Y. Moses, "Coordinated consensus in dynamic networks," Acm Symposium on Principles of Distributed Computing 1-10, 2011.

[23] S.K. Maity, T.V. Manoj, and A. Mukherjee, "Opinion dynamics in correlated time-varying social networks," Social Networks, Datasets, Hypertext / Working Paper.

[24] R. Olfati-Saber, and R.M. Murray, "Consensus problems in networks of agents with switching topology and time-delays,"IEEE Transactions on Automatic Control 49, 1520-1533, 2015.

[25] R.Y. Tian, and Y.J. Liu, "Isolation, insertion, and reconstruction: Three strategies to intervene in rumor spread based on super-network model," Decision Support Systems 67,121-130, 2014.

[26] A. Mislove, M. Marcon, K.P. Gummadi, P. Druschel, and B. Bhattacharjee, "Measurement and analysis of online social networks," Acm Sigcomm Conference on Internet Measurement 71, 1815-1816, 2007.

[27] S. Patterson, and B. Bamieh, "Interaction-driven opinion dynamics in online social networks," Workshop on Social Media Analytics, 98-105, 2010.

[28] J.A. Bondy, and U.S.R. Murty, Graph Theory with Applications. The Macmillan press LTD, 1976

[29] R.A. Horn, and C. R. Johnson, Matrix Analysis (2nd ed.), Cambridge University Press, 1985.

[30] R. Diestel, Graph Theory (3rd ed.), Springer, 2005

[31] J. Wolfowitz, "Products of Indecomposable, Aperiodic, Stochastic Matrices," Proceedings of the American Mathematical Society 14,733733, 1963.

[32] A. Jadbabaie, J. Lin, and A.S. Morse, "Coordination of groups of mobile autonomous agents using nearest neighbor rules," IEEE Transactions on Automatic Control 48, 988-1001, 2015.

[33] M. E. J. Newman, The structure and function of complex networks, Siam Review 45, 167-256, 2006.

[34] Erdős, Paul, and A. Rényi, "On the evolution of random graphs," Publication of the Mathematical Institute of the Hungarian Academy Ofences 38, 17-61, 1960. 
[35] D.J. Watts, and S.H. Strogatz, "Collective dynamics of 'small-world' networks," Nature 393(6684), 440-442, 1998.

[36] Barabási, Albert-László, and Albert Réka, "Emergence of scaling in random networks," Science 286 (5439), 509-512, 1999.

[37] S. Peng, A. Yang, L. Cao, S. Yu, and D. Xie, "Social Influence modeling using information theory in mobile social networks," Information Sciences, 1-14, 2016.

[38] P. Victor, C. Cornelis, M. De Cock, and E. Herrera-Viedma, "Practical Aggregation Operators for Gradual Trust and Distrust," Fuzzy Sets and Systems 184(1), 126-147, 2011.

[39] N. Verbiest, C. Cornelis, P. Victor, and E. Herrera-Viedma, "Trust and distrust aggregation enhanced with path length incorporation," Fuzzy Sets and Systems 202(1), 61-74, 2012.

[40] J. Wu, F. Chiclana, E. Herrera-Viedma, "Trust Based Consensus Model for Social Network in an Incomplete Linguistic Information Context," Applied Soft Computing 35, 827-839, 2015.

[41] Y. Dong, H. Zhang, and E. Herrera-Viedma, "Integrating experts' weights generated dynamically into the consensus reaching process and its applications in managing non-cooperative behaviors," Decision Support Systems 84, 1-15, 2016.

[42] W. Liu, Y. Dong, F. Chiclana, F.J. Cabrerizo, and E. Herrera-Viedma, "Group decision-making based on heterogeneous preference relations with self-confidence," Fuzzy Optimization and Decision Making, in press. Doi: 10.1007/s10700-016-9254-8, 2017.

[43] L. Fang, P.J. Antsaklis, and A. Tzimas, "Asynchronous consensus protocols: preliminary results, simulations and open questions," European Control Conference Cdc-ecc 05 IEEE Conference on Decision \& Control, 2194-2199, 2005.

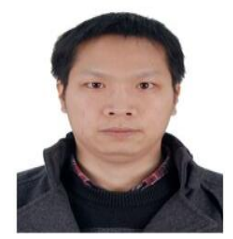

Yucheng Dong is a Professor at the Business School, Sichuan University, China. He received his Ph.D. degree in management from Xi'an Jiaotong University in 2008. His current research interests include group decision making, decision support systems, computing with words in decision making, and and opinion dynamics. Dr. Dong published over 60 international journal papers in Decision Support Systems, European Journal of Operational Research, IEEE Transactions on Cybernetics, IEEE Transactions on Fuzzy Systems, IEEE Transactions on Systems, Man, and Cybernetics, among others. Several of his papers (currently 10) are classed as highly cited by ISI Web of Science. Dr. Dong is a member of the editorial board of the journal Information Fusion.

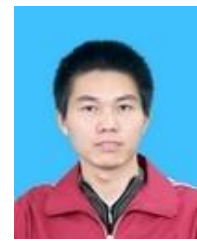

Zhaogang Ding is a Ph.D. candidate at the Business School, Sichuan University, China. He received his B.S. degree from Zhengzhou University in 2009 and M.S. degree from Wuhan University in 2011. His research interests include opinion dynamics, social network analysis and collective action.

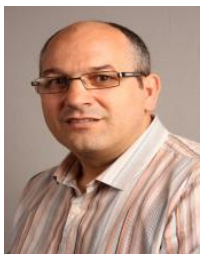

Professor Francisco Chiclana received the B.Sc. and Ph.D. degrees in Mathematics, both from the University of Granada (Spain) in 1989 and 2000, respectively. He is currently a Professor of Computational Intelligence and Decision Making at De Montfort University (Leicester, UK). He is Associate Editor of Soft Computing Journal and the Journal of Intelligent and Fuzzy Systems, and member of the editorial board of the ISI journals Information Sciences, Applied Soft Computing, Knowledge-Based Systems, MultipleValued Logic and Soft Computing, and Journal of Applied Mathematics. He has published extensively in leading international journals such as: IEEE TFS; IEEE TSMC (Part A/Part B); EJOR; FSS; INS;
IJAR; KNOSYS, IJIS and ASOC. Several of his papers (currently 16) are classed as highly cited. He has also been consistently classed as one of the Most Cited Scientist in its field. His current (September 2014) 75 records in ISI WoK have received a total of 3152 citations, with an h-index of 24 .

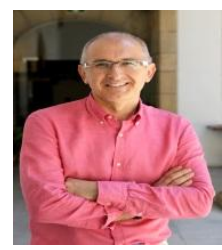

Enrique Herrera-Viedma is a Professor of Computer Science and the Vice-President for Research and Transfer in University of Granada. He received the M.Sc. and Ph.D. degrees in Computer Science from the University of Granada in 1993 and 1996, respectively. His current research interests include group decision making, consensus models, linguistic modelling, and aggregation of information, information retrieval, bibliometric, digital libraries, web quality evaluation, recommender systems, and social media. His hindex is 48 with more than 7500 citations received [WoS]. He was identified in 2014 and 2015 as one of the world's most influential researchers by the Shanghai Center and Thomson Reuters in both Computer Science and Engineering. Dr. Herrera-Viedma is an Associate Editor of several journals such as IEEE Transactions on Systems Man and Cybernetics: Systems, Information Sciences, and Knowledge-Based Systems. 